\title{
Dynamics of photodoped charge transfer insulators
}

\author{
Denis Golež ${ }^{1,2}$ Lewin Boehnke, ${ }^{1, *}$ Martin Eckstein, ${ }^{3}$ and Philipp Werner ${ }^{1}$ \\ ${ }^{1}$ Department of Physics, University of Fribourg, 1700 Fribourg, Switzerland \\ ${ }^{2}$ Center for Computational Quantum Physics, Flatiron Institute, 162 Fifth Avenue, New York, NY 10010, USA \\ ${ }^{3}$ Department of Physics, University of Erlangen-Nürnberg, 91058 Erlangen, Germany
}

(Received 7 August 2018; revised manuscript received 21 January 2019; published 17 July 2019)

\begin{abstract}
We study the dynamics of charge transfer insulators after photoexcitation using the three-band Emery model and a nonequilibrium extension of Hartree-Fock + EDMFT (extended dynamical mean field theory) and $G W+$ EDMFT. While the equilibrium properties are accurately reproduced by the Hartree-Fock treatment of the ligand bands, dynamical correlations are essential for a proper description of the photodoped state. Photodoping leads to a renormalization of the charge transfer gap and to a substantial broadening of the bands. We calculate the time-resolved photoemission spectrum and optical conductivity and find qualitative agreement with experiments. Our formalism enables the realistic description of nonequilibrium phenomena in materials with ligand bands. It provides a tool to explore the optical manipulation of interaction and correlation effects, including insulator-metal and magnetic transitions.
\end{abstract}

DOI: 10.1103/PhysRevB.100.04111

The ability to engineer the microscopic parameters of correlated electron materials is important for the understanding of their complex properties and for technological applications. An example is the control of superconductivity by pressure, doping, or strain [1-3] under equilibrium conditions. Recent progress in ultrafast laser techniques motivates new strategies which involve nonthermal states. Experiments have demonstrated, e.g., the switching to a hidden metallic state in 1T$\mathrm{TaS}_{2}$ [4], or light-induced enhancements of superconductivity [5,6] and excitonic order [7]. Related are theoretical proposals to modify the band structure $[8,9]$ or interactions by electronic excitations [10-12] or phonon driving [13-19].

A simple way to change the properties of correlated materials is the photoexcitation of charge carriers across a Mott or charge transfer (CT) gap. Most previous theoretical nonequilibrium studies of Mott insulators have focused on single-band models [20-27], which miss important aspects of the physics of CT insulators with $p$ and $d$ bands. In these compounds, photoexcitation results in doublon and holon charge carriers of a qualitatively different nature, and depending on the excitation energy one may selectively excite electrons from occupied $p$ or $d$ states. Photodoping also provides a means to change the relative position of the $p$ and $d$ bands (band-gap renormalization). Since the charge transfer gap affects the insulating and magnetic properties of these materials, this offers a playground for photomanipulation of these phases.

A paradigmatic class of $\mathrm{CT}$ insulators are the cuprates. The equilibrium properties of cuprates have been investigated with a broad range of methods including exact diagonalization [28,29], dynamical mean field theory (DMFT) [30-32], and its cluster extensions [33]. These studies emphasized the importance of a multiband description including $p$ orbitals [34-36] and the role of spin [33] and charge fluctuations

*Now at Crypto Finance AG
$[37,38]$. While in equilibrium the correlation effects in the full ligand bands are weak, photodoping induces charge carriers and nontrivial correlations. In fact, pump-probe studies on cuprate superconductors have detected photoinduced band shifts and modifications in effective masses [39-44], as well as a redistribution of spectral weight between different characteristic energy scales $[45,46]$.

In this work, we consider the two-dimensional three-band Emery model [47] describing the $\mathrm{Cu} d_{x^{2}-y^{2}}$ (denoted $d$ ) and $O p_{x}$ and $p_{y}$ orbitals, where the $O$ orbitals lie between $\mathrm{Cu}$ ions forming a square lattice with lattice constant $a$. The three terms of the Hamiltonian $H=H_{\mathrm{e}}+H_{\text {kin }}+H_{\text {int }}$ are

$$
\begin{aligned}
H_{\mathrm{e}} & =\epsilon_{d} \sum_{i} n_{i}^{d}+\left(\epsilon_{d}+\Delta_{p d}\right) \sum_{i}\left(n_{i}^{p_{x}}+n_{i}^{p_{y}}\right), \\
H_{\mathrm{kin}} & =\sum_{i j \sigma} \sum_{(\alpha, \beta) \in\left(d, p_{x}, p_{y}\right)} t_{i j}^{\alpha \beta} c_{i \alpha \sigma}^{\dagger} c_{j \beta \sigma}, \\
H_{\mathrm{int}} & =\sum_{i j} \sum_{(\alpha, \beta) \in\left(d, p_{x}, p_{y}\right)} V_{i j}^{\alpha \beta} n_{i}^{\alpha} n_{j}^{\beta},
\end{aligned}
$$

with $V_{i j}^{\alpha \beta}=U_{d d}$ if $i=j$ and $\alpha=\beta=d$, and $V_{i j}^{\alpha \beta}=\frac{1}{2} U_{d p}$ for nearest-neighbor $d$ and $p$ orbitals. The crystal field splitting $\Delta_{d p}$ determines the difference between the on-site energy for the $d$ orbital, $\epsilon_{d}$, and that for the $p$ orbitals, $\epsilon_{d}+\Delta_{d p}$. We denote the nearest-neighbor hopping between the $d$ and $p_{x}$, $p_{y}$ orbitals by $t^{d p}$ and between the $p_{x}$ and $p_{y}$ orbitals by $t^{p p}$, while $-t_{i i}^{\alpha \alpha}=\mu$ is the chemical potential.

We solve this lattice problem using the fully selfconsistent $G W+$ EDMFT [11,48-50] and Hartree-Fock (HF) + EDMFT methods, which are based on extended dynamical mean field theory (EDMFT) [10,49-51], and a noncrossing approximation impurity solver [10]. Since the local interactions in the $d$ orbital are stronger than in the $p$ orbitals, we restrict the EDMFT treatment to the $d$ orbital, while the $p$ orbitals are treated at the $\mathrm{HF}$ or $G W$ level. 


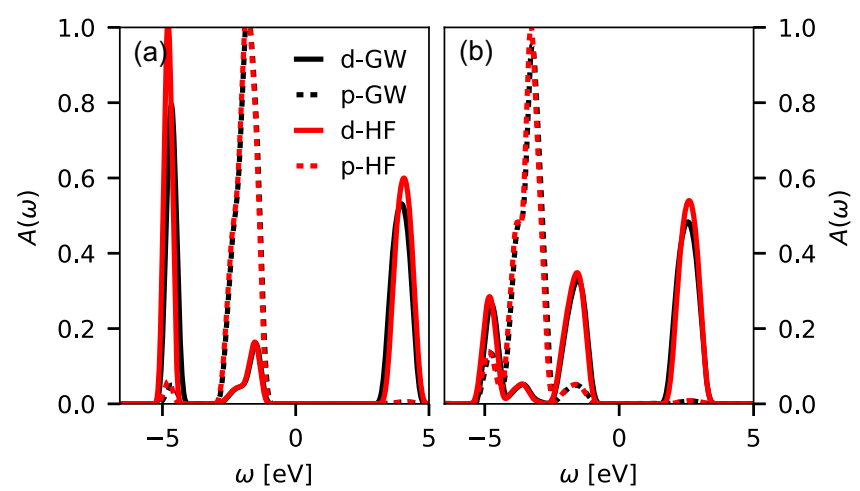

FIG. 1. Orbitally resolved spectral function obtained in the $G W+$ EDMFT (black) and HF + EDMFT (red) approximation for the CT (a) and LCO (b) setups. The full (dashed) lines represent the $d\left(p_{x}\right.$ and $\left.p_{y}\right)$ orbitals.

The dynamics is described in terms of the momentum and orbital-resolved electronic Green's function $G_{k \sigma}^{\alpha \beta}\left(t, t^{\prime}\right)=$ $-i\left\langle\mathcal{T}_{\mathcal{C}} c_{k \alpha \sigma}(t) c_{k \beta \sigma}^{\dagger}\left(t^{\prime}\right)\right\rangle$ and the charge correlation function $\chi_{q}^{\alpha \beta}\left(t, t^{\prime}\right)=-i\left\langle T_{\mathcal{C}} \tilde{n}_{q \alpha}(t) \tilde{n}_{-q \beta}\left(t^{\prime}\right)\right\rangle$, which determines the inverse of the orbital-resolved dielectric function $\left[\varepsilon_{q}^{-1}\right]=I+$ $v_{q} * \chi_{q}$ and the screened interaction $W_{q}=\left[\varepsilon_{q}^{-1}\right] * v_{q}$, where $v_{q}$ is the Fourier transform of the bare interaction. The mapping of the lattice problem to an impurity problem and the downfolding procedure lead to a retarded density-density interaction $\mathcal{U}\left(t, t^{\prime}\right)$ on the impurity ( $d$ orbital), which is related to the screened interaction by $W_{\text {loc }}^{d d}=\mathcal{U}+\mathcal{U} * \chi_{\text {imp }} * \mathcal{U}$.

The electromagnetic field leads to an acceleration of the charge carriers as well as dipolar transitions. The gauge invariant description of both effects is given by the modified hopping term [52,53]

$$
H_{\text {kin }}=\sum_{\substack{i j \sigma,(\alpha, \beta) \\ \epsilon\left(d, p_{x}, p_{y}\right)}}\left(t_{i j}^{\alpha \beta}+\vec{E} \cdot \vec{D}_{i j}^{\alpha \beta}\right) e^{-i \varphi_{i j}} c_{i \alpha \sigma}^{\dagger} c_{j \beta \sigma},
$$

where $\varphi_{i j}=e / \hbar \int_{R_{i}}^{R_{j}} d \vec{r} \vec{A}(\vec{r}, t)$ is the Peierls phase and $\vec{A}(\vec{r}, t)$ is the vector potential, which is related to the electric field by $\vec{E}(\vec{r}, t)=-\partial_{t} \vec{A}(\vec{\rho}, t)$. We assume that $\vec{A}(t)$ and $\vec{E}(t)$ are homogeneous in space. For a quantitative description of the photoexcitation in multiband materials the dipolar matrix elements have to be obtained from $a b$ initio calculations. In the following simulations, we use nearest neighbor $d$ to $p$ dipolar matrix elements $D \equiv D_{(i,\{0,0\})(i,\{a / 2,0\})}^{p_{x} d}=-D_{(i,\{0,0\})(i,\{a / 2,0\})}^{p_{y} d}=$ $0.16 e a[54]$.

We will study two characteristic setups previously considered in the literature: (i) a charge transfer insulator close to the atomic limit, where a strong Coulomb repulsion opens a large Mott gap and narrow $p$ bands lie well separated between the lower and the upper Hubbard bands ("CT case"), and (ii) the parameter regime relevant for $\mathrm{La}_{2} \mathrm{CuO}_{4}$, as obtained from local density approximation (LDA) calculations and parametrized by a tight-binding Hamiltonian (LCO case) following Refs. [37] and [34]. In this setup the $p$ bands lie in the same energy range as the lower Hubbard band.

In Fig. 1 we present the orbitally resolved local spectral functions $A_{\alpha}(\omega)=-\frac{1}{\pi} \operatorname{Im}\left[G_{\mathrm{loc}}^{\alpha, \alpha}(\omega)\right]$, for $\alpha=d, p_{x}, p_{y}$. In all calculations we set the inverse temperature to $\beta=5.0 \mathrm{eV}^{-1}$,

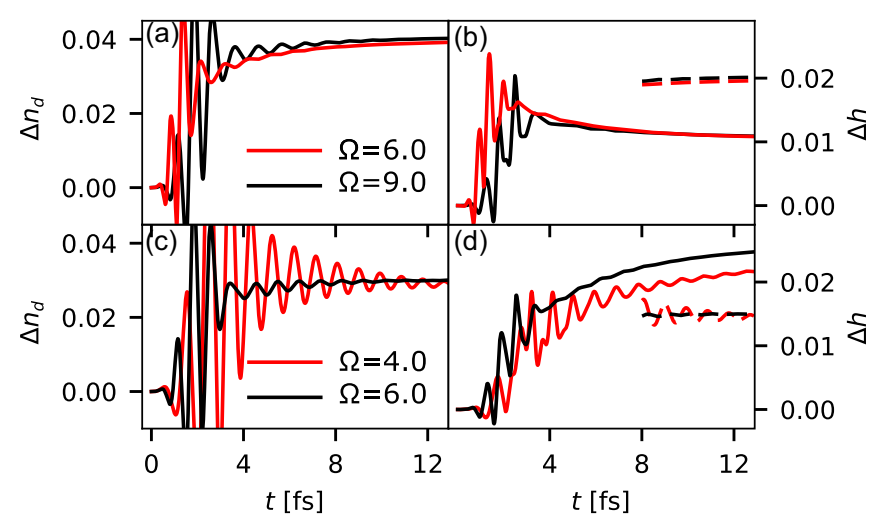

FIG. 2. Dynamics after photoexcitation for the CT (top panels) and LCO (bottom panels) setups. The left panels show the change in the occupancy of the $d$ orbitals. The change in the $p$ orbital occupation is given by $\Delta n_{p}=-\frac{1}{2} \Delta n_{d}$. The right panel shows the change in the density of $d$ holes $\Delta h_{d}$ in the lower Hubbard band. The hole density on the $p$ orbital can be estimated as $\Delta h_{p}=\frac{1}{2} \Delta n_{d}$ and is shown by the dashed lines in the time interval [8:13] for clarity.

which is above the Néel temperature. In the $\mathrm{CT}$ case [Fig. 1(a)], with parameters $U_{d d}=8.0 \mathrm{eV}, U_{d p}=2.0 \mathrm{eV}$, $t_{d p}=0.4 \mathrm{eV}, t_{d d}=-0.1 \mathrm{eV}, t_{p p}=0.15 \mathrm{eV}, \Delta_{p d}=-2.0 \mathrm{eV}$, the lower Hubbard band is well separated from the $p$ bands. In the example relevant for $\mathrm{La}_{2} \mathrm{CuO}_{4}$ [Fig. 1(b)], with parameters $U_{d d}=5.0 \mathrm{eV}, U_{d p}=2.0 \mathrm{eV}, t_{d p}=0.5 \mathrm{eV}, t_{d d}=-0.1 \mathrm{eV}$, $t_{p p}=0.15 \mathrm{eV}, \Delta_{p d}=-3.5 \mathrm{eV}$, the spectral function below the Fermi level is split into three distinct peaks: a peak at $\omega=$ $-3.3 \mathrm{eV}$ of predominantly $p$-orbital character, the antibonding band corresponding to the Zhang-Rice singlet (ZR) around $\omega=-1.6 \mathrm{eV}[32,33,55]$, and the bonding band pushed to lower energy, with a center at $\omega=-4.9 \mathrm{eV}$. In equilibrium, the $G W+$ EDMFT and HF + EDMFT spectra are almost indistinguishable, which shows that static correlations are sufficient for the treatment of the fully occupied $p$ orbitals. In a chemically doped system this is not any more the case and we expect nonlocal fluctuations to become important. We also note that the gap reduces the effect of screening on the local interaction due to a suppression of charge fluctuations [56]. As a result, the effective static interaction is only slightly lower than the bare interaction: $\mathcal{U}(\omega=0)-U_{d d} \approx-0.03 \mathrm{eV}$ for the CT and $-0.05 \mathrm{eV}$ for the LCO setup.

We now turn to the photoexcitation and the subsequent relaxation. Due to the Mott physics, the equilibrium occupation of the $d$ band is half-filled, while the $p$ bands are completely filled. A short pulse $E(t)=E_{0} e^{-4.6\left(t-t_{0}\right)^{2} / t_{0}^{2}} \sin \left[\Omega\left(t-t_{0}\right)\right]$ polarized along the (11) direction transfers charge from $p$ to $d$ (see Fig. 2). The width of the pulse $t_{0}=2 \pi n / \Omega$ is chosen such that the envelope accommodates $n=2$ cycles. Due to the mixed nature of the states below the Fermi level one may expect that the ratio between the excited electrons originating from the $p$ or $d$ orbital depends on the frequency of the pulse. In the following we will choose frequencies corresponding to a photoexcitation from the characteristic features in the occupied part of the spectrum to the upper Hubbard band. The excitation strength $E_{0}$ at each given frequency is adjusted such that the density of photodoped doublons and holons is approximately 5\%. Due to the large gap size this 
density changes only very slowly after the photoexcitation $[24,27,57,58]$.

In Fig. 2(a) we present the time-dependent change in the $d$ orbital occupancy $n_{d}$ for the CT setup and two different frequencies corresponding to the transitions from the $p$ band $\left(\Omega=6 \mathrm{eV}, E_{0}=0.37 \mathrm{eV} / a\right)$ and the lower Hubbard band $\left(\Omega=9 \mathrm{eV}, E_{0}=0.87 \mathrm{eV} / a\right)$ to the upper Hubbard band. While the change in the double occupancy $d_{\text {occ }}$ corresponds to the total amount of charge transferred across the gap, the change in the density of holes in the lower Hubbard band corresponds to $\Delta h_{d}=\Delta d_{\mathrm{occ}}-\Delta n_{d}$, which is plotted in Fig. 2(b). The final occupations are remarkably independent of the pulse frequency. The spectrally resolved occupation (not shown) indicates that after an ultrafast redistribution of occupation between the region of the lower Hubbard band and the $p$ band the holes predominantly reside in the $p$ band. This rapid interband decay is only observed within the $G W+$ DMFT formalism and not in HF + DMFT, so that its likely origin is the strong coupling of electrons to charge fluctuations [11].

In the LCO case we excite electrons to the upper Hubbard band either from the ZR singlet $\left(\Omega=4, E_{0}=0.54 \mathrm{eV} / a\right)$ or from the band with dominant $p$ character $\left(\Omega=6.0, E_{0}=\right.$ $0.31 \mathrm{eV} / a$ ). Due to the smaller gap size, impact-ionization processes become important and lead to a more rapid increase of the double occupancy after the photoexcitation [59]. For a fixed number of doubly occupied sites there is a larger percentage of holes in the $d$ orbitals than for the CT insulator [see Figs. 2(b) and 2(d)]. The holes predominantly occupy the ZR band as seen in the greater component of the orbitally resolved spectral function (not shown). Apart from strong initial oscillations due to the $p-d$ hybridization the final occupation is again rather weakly dependent on $\Omega$.

We next discuss the time evolution of the spectrum after the photoexcitation by analyzing the partial Fourier transform of the orbitally resolved spectral function $A_{\alpha}(\omega, t)=$ $-(1 / \pi) \operatorname{Im}\left[\int_{t}^{t+t_{\text {cut }}} d t^{\prime} e^{i \omega\left(t^{\prime}-t\right)} G_{\alpha}^{R}\left(t^{\prime}, t\right)\right]$, with $t_{\text {cut }}=8$ and $\alpha \in$ $\left\{d, p_{x}, p_{y}\right\}$. Again we will compare the $G W+$ EDMFT and HF + EDMFT approximations in order to address the role of nonlocal charge fluctuations. Due to the Coulomb interaction $U_{d p}$ between the holes in the $p$ orbitals and the doublons in the $d$ orbital there is an almost instantaneous reduction of the gap between the $p$ band and the upper Hubbard band (see Fig. 3). It originates from the static Hartree-Fock attraction and is determined by the change in the local orbital occupancy $\Delta \Sigma_{d d}^{\mathrm{H}}=\left(U_{d d}-2 U_{d p}\right) \Delta n_{d}[44,60,61]$. The contribution of this effect to the shrinking gap is indicated in Fig. 3 by the vertical red line, $\Delta \Sigma_{d d}^{\mathrm{H}}=-0.15(-0.17) \mathrm{eV}$ for the $\mathrm{CT}$ (LCO) case.

Going beyond the HF description, the inclusion of nonlocal charge fluctuations leads to (a) a further reduction of the gap size due to an additional band shift, and (b) a substantial broadening of the spectra (in particular for the $d$ orbitals) due to a strong electron-plasmon coupling (see Fig. 3). Both effects are a consequence of the photoinduced changes in the screening. The additional gap shrinking can be quantified by the reduced static interaction, $\mathcal{U}(\omega=0)-U_{d d} \approx-0.14 \mathrm{eV}$ for both setups, which is indicated by the green vertical line in Fig. 3. One can see that the full reduction of the gap cannot be described by these static contributions alone

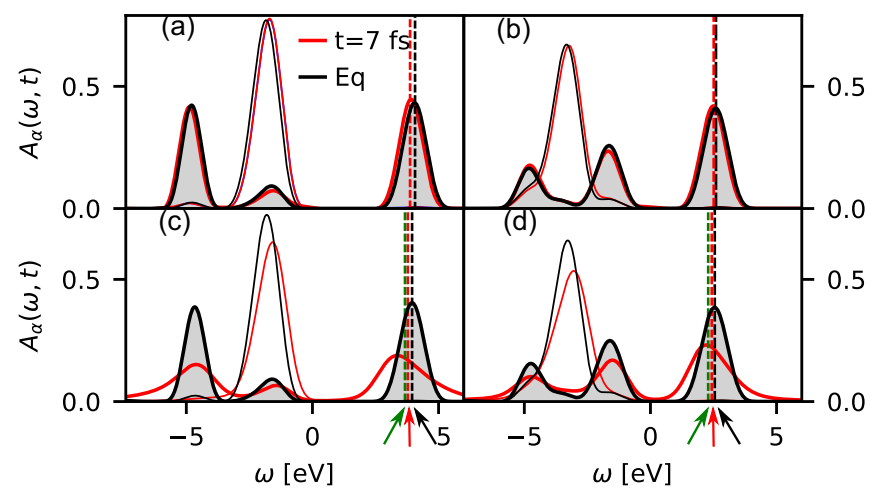

FIG. 3. Time evolution of the spectral function $A_{\alpha}(\omega, t)$ within $\mathrm{HF}+\operatorname{EDMFT}[(\mathrm{a})$ and (b)] and $G W+\operatorname{EDMFT}[(\mathrm{c})$ and (d)] for the CT (left panels) and LCO (right panels) setups, both excited with the pulse frequency $\Omega=6.0 \mathrm{eV}$. The black lines show the equilibrium spectra. Thick shaded (thin) lines represent the $d$ ( $p_{x}$ and $p_{y}$ ) orbitals. The black vertical line and the arrow mark the peak position of the upper Hubbard band in equilibrium, the red ones indicate the Hartree shift, and the green ones correspond to the combined effect of Hartree shift and static reduction of the effective impurity interaction $\mathcal{U}(\omega=0)$.

and must be attributed to dynamical screening and doping effects. Comparing Fig. 3 with Fig. 1 we conclude that the treatment of both dynamic screening and the feedback of the nonlocal charge fluctuations is qualitatively important for the description of the photoexcited state, while these effects are negligible in equilibrium [62].

The dynamics of the $p$ band exhibits a remarkable resemblance with recent time-resolved angle-resolved photoemission spectroscopy data, which for optimally doped Y-Bi2212 show a nonthermal broadening of the $2 p_{\pi}$ band at momentum $(\pi, \pi)$ [44]. The interpretation of Ref. [44], which attributed the nonthermal changes in the experimental spectra mainly to nonlocal charge fluctuations on top of a minor static Hartree shift, is fully consistent with our results.

The information about the time-dependent changes in the screening properties of the $d$ orbital is contained in the screened interaction $W_{d}(\omega, t)$ (see Fig. 4). The equilibrium screening modes for the charge transfer insulator include (i) a peak at $\omega_{C T} \approx 6 \mathrm{eV}$ corresponding to particle-hole excitations from the $p$ band to the upper Hubbard band, and

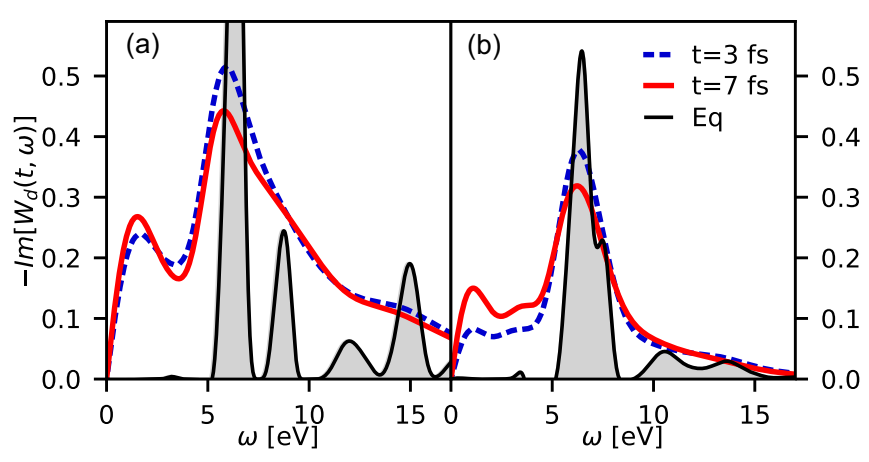

FIG. 4. Time evolution of the screened interaction $W_{d}(t, \omega)$ for the CT (a) and LCO (b) setups, both excited with frequency $\Omega=$ $6.0 \mathrm{eV}$. The black shaded line represents the equilibrium spectrum. 
(ii) a peak at $\omega_{H} \approx 8.5 \mathrm{eV}$ matching the excitations from the lower to the upper Hubbard band, and higher order excitations [see also Fig. 1(a)]. After the photoexcitation these features are smeared out and a continuum of screening modes appears at low energies. These are associated with charge excitations within the photodoped bands and result in an additional screening [10]. Note that this is a unique nonequilibrium feature of the electron-plasmon dynamics and does not have a counterpart in couplings to other bosonic degrees of freedom, like phonons. Within EDMFT one can define an effective coupling of the electrons and the charge fluctuations from the integral over $\operatorname{Im} \mathcal{U}(\omega) / \omega$ [or, equivalently, the reduction of $\operatorname{Re} \mathcal{U}(\omega=0)][10,11,49]$. The coupling strength to the photoinduced charge fluctuations at $\omega \lesssim$ $6 \mathrm{eV}, \lambda_{\text {ind }}=2 \int_{0}^{6} d \omega(-\operatorname{Im}[\mathcal{U}(\omega)] / \omega) \approx 0.16$, corresponds to a rather strong coupling, which explains the substantial broadening of the spectra in Fig. 3. The dynamics of $W_{d}(t, \omega)$ in the LCO case is qualitatively similar except for an additional peak at $\omega \approx 3.5$, which originates from the charge excitations between the Zhang-Rice singlet and the lower edge of the upper Hubbard band. The effective electron-plasmon coupling $\lambda_{\text {ind }} \approx 0.17$ is comparable to the $\mathrm{CT}$ case.

Finally, we discuss the signature of the band-gap renormalization in the optical conductivity. We explicitly simulate a probe pulse and extract the photoinduced current as the difference in the current with and without a probe pulse, $j_{\text {probe }}=j_{\text {pump }+ \text { probe }}-j_{\text {pump }}$. For a weak probe pulse the optical conductivity can be evaluated as the ratio $\sigma\left(\omega, t_{p}\right)=$ $j\left(\omega, t_{p}\right) / E\left(\omega, t_{p}\right)$, where $X\left(\omega, t_{p}\right)=\int_{0}^{t_{\text {cut }}} d s X(t+s) e^{-i \omega s}$ is the Fourier transform of $X=j, E$, and $t_{p}$ is the center of the probe pulse. This procedure avoids the calculation of the current-current correlation function including vertex corrections. We apply both the pump and probe pulses in the (11) direction.

The optical conductivity in equilibrium (black lines) and for $t_{p}=7 \mathrm{fs}$ after the pump pulse is presented in Figs. 5(a) and 5(c). The equilibrium optical conductivity for both cases exhibits a main peak corresponding to excitations from the $p$ band to the upper Hubbard band. In the LCO case [see Fig. 5(c)] the small peak at $\omega=4.4 \mathrm{eV}$ corresponds to excitations from the ZR singlet to the upper Hubbard band.

After the photoexcitation the optical conductivity shows a clear shift toward lower energies, originating from the HF shift and the enhanced screening. In order to highlight this evolution we also plot the change of the optical conductivity from the equilibrium value [Fig. 5(b)] as is usually done in the experimental literature [43]. Indeed, a characteristic shift of the excitation gap to lower energies (redshift) has been reported in a number of pump-probe experiments on cuprates and other charge transfer insulators (see, for instance, Refs. [40-43,63]). The comparison of the shift to the static screening contribution [line labeled "Stat" in Fig. 5(d)] shows that more than $50 \%$ of the gap renormalization is due to dynamical effects.

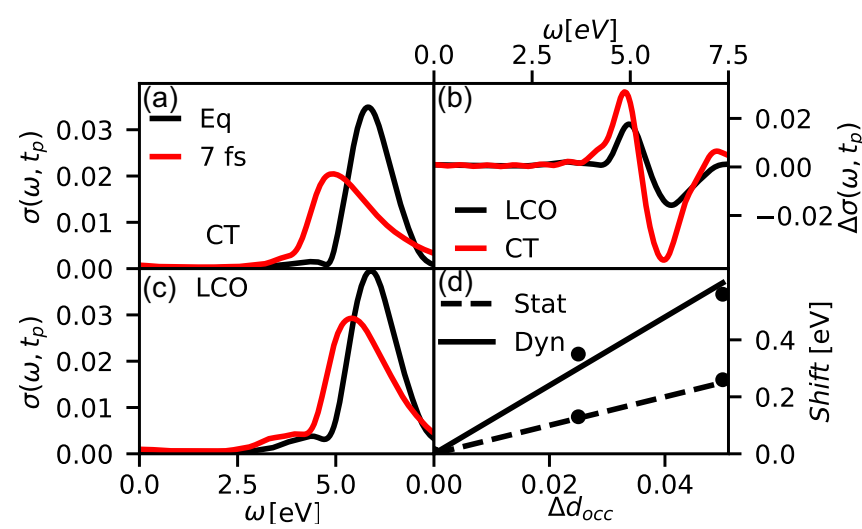

FIG. 5. Time-dependent optical conductivity in the (11) direction $\sigma\left(\omega, t_{p}\right)$ for the CT (a) and LCO (c) setups. The black lines represent the equilibrium data. (b) The difference from the equilibrium result $\Delta \sigma\left(t_{p}, \omega\right)=\sigma\left(t_{p}, \omega\right)-\sigma^{\mathrm{eq}}(\omega)$ for the delayed probe pulse at $t_{p}=$ $7 \mathrm{fs}$ in both setups. (d) The gap size renormalization in the optical conductivity (Dyn) as a function of the photoexcited doublons and comparison with the static shift (Stat) $\Sigma_{H}+\mathcal{U}(\omega=0)$.

A recent experimental study of photodoped $\mathrm{La}_{2} \mathrm{CuO}_{4}$ [43] reports an even somewhat larger shift than predicted by the line labeled "Dyn" in Fig. 5(d). This suggests that dynamical effects, captured by the $G W+$ EDMFT method, are essential for a quantitative description of photodoped cuprates.

In conclusion, we have studied the pump-probe dynamics of the three-band Emery model, relevant for a large family of charge transfer insulators. The electric field pulse transfers charge from the $p$ bands to the upper Hubbard band, which results in relative band shifts. Similar band shifts have been observed in a recent time-dependent density functional theory (TDDFT) $+U$ study of $\mathrm{NiO}$ [12], while the present study shows that charge fluctuations strongly enhance this effect. The strong plasmon coupling in the photodoped state leads to a substantial broadening of the $d$ and $p$ bands, which implies that dynamical correlation effects (beyond HF) are essential for the description of the ligand bands, in contrast to equilibrium.

Our work represents a crucial step toward the ab initio description of strongly correlated systems out of equilibrium, where the material-specific input is obtained via a multitier approach analogous to the scheme recently demonstrated for equilibrium systems in Refs. [37,64,65].

We thank Y. Murakami and A. J. Millis for useful discussions, and M. Schüler for advice on computational issues. The calculations have been performed on the Beo04 and Beo05 clusters at the University of Fribourg. D.G. and P.W. acknowledge support from SNSF Grant No. 200021_165539 and ERC Consolidator Grant No. 724103. M.E. acknowledges financial support from ERC Starting Grant No. 716648. The Flatiron Institute is a division of the Simons Foundation.
[1] B. Sipos, A. F. Kusmartseva, A. Akrap, H. Berger, L. Forró, and E. Tutiš, Nat. Mater. 7, 960 (2008).
[2] R. H. Zadik, Y. Takabayashi, G. Klupp, R. H. Colman, A. Y. Ganin, A. Potočnik, P. Jeglič, D. Arčon, P. Matus, K. Kamarás, 
Y. Kasahara, Y. Iwasa, A. N. Fitch, Y. Ohishi, G. Garbarino, K. Kato, M. J. Rosseinsky, and K. Prassides, Sci. Adv. 1, e1500059 (2015).

[3] O. Ivashko, M. Horio, W. Wan, N. Christensen, D. McNally, E. Paris, Y. Tseng, N. Shaik, H. Rønnow, H. Wei et al., Nat. Commun. 10, 786 (2019).

[4] L. Stojchevska, I. Vaskivskyi, T. Mertelj, P. Kusar, D. Svetin, S. Brazovskii, and D. Mihailovic, Science 344, 177 (2014).

[5] D. Fausti, R. I. Tobey, N. Dean, S. Kaiser, A. Dienst, M. C. Hoffmann, S. Pyon, T. Takayama, H. Takagi, and A. Cavalleri, Science 331, 189 (2011).

[6] M. Mitrano, A. Cantaluppi, D. Nicoletti, S. Kaiser, A. Perucchi, S. Lupi, P. Di Pietro, D. Pontiroli, M. Riccò, S. R. Clark et al., Nature (London) 530, 461 (2016).

[7] S. Mor, M. Herzog, D. Golež, P. Werner, M. Eckstein, N. Katayama, M. Nohara, H. Takagi, T. Mizokawa, C. Monney, and J. Stähler, Phys. Rev. Lett. 119, 086401 (2017).

[8] T. Oka and H. Aoki, Phys. Rev. B 79, 081406(R) (2009).

[9] N. Tsuji, T. Oka, P. Werner, and H. Aoki, Phys. Rev. Lett. 106, 236401 (2011).

[10] D. Golež, M. Eckstein, and P. Werner, Phys. Rev. B 92, 195123 (2015).

[11] D. Golež, L. Boehnke, H. U. R. Strand, M. Eckstein, and P. Werner, Phys. Rev. Lett. 118, 246402 (2017).

[12] N. Tancogne-Dejean, M. A. Sentef, and A. Rubio, Phys. Rev. Lett. 121, 097402 (2018).

[13] M. Knap, M. Babadi, G. Refael, I. Martin, and E. Demler, Phys. Rev. B 94, 214504 (2016).

[14] M. Babadi, M. Knap, I. Martin, G. Refael, and E. Demler, Phys. Rev. B 96, 014512 (2017).

[15] M. A. Sentef, Phys. Rev. B 95, 205111 (2017).

[16] Y. Murakami, N. Tsuji, M. Eckstein, and P. Werner, Phys. Rev. B 96, 045125 (2017).

[17] G. Mazza and A. Georges, Phys. Rev. B 96, 064515 (2017).

[18] D. M. Kennes, E. Y. Wilner, D. R. Reichman, and A. J. Millis, Nat. Phys. 13, 479 (2017).

[19] Y. Murakami, D. Golež, M. Eckstein, and P. Werner, Phys. Rev. Lett. 119, 247601 (2017).

[20] H. Aoki, N. Tsuji, M. Eckstein, M. Kollar, T. Oka, and P. Werner, Rev. Mod. Phys. 86, 779 (2014).

[21] M. Eckstein and P. Werner, Sci. Rep. 6, 21235 (2016).

[22] M. Eckstein and P. Werner, Phys. Rev. Lett. 113, 076405 (2014).

[23] M. Eckstein and P. Werner, Phys. Rev. Lett. 110, 126401 (2013).

[24] M. Eckstein and P. Werner, Phys. Rev. B 84, 035122 (2011).

[25] D. Golež, J. Bonča, M. Mierzejewski, and L. Vidmar, Phys. Rev. B 89, 165118 (2014).

[26] S. Dal Conte, L. Vidmar, D. Golež, M. Mierzejewski, G. Soavi, S. Peli, F. Banfi, G. Ferrini, R. Comin, B. M. Ludbrook et al., Nat. Phys. 11, 421 (2015).

[27] Z. Lenarčič and P. Prelovšek, Phys. Rev. Lett. 111, 016401 (2013).

[28] J. Jaklič and P. Prelovšek, Adv. Phys. 49, 1 (2000).

[29] T. Tohyama, Phys. Rev. B 70, 174517 (2004).

[30] A. Comanac, L. de'Medici, M. Capone, and A. Millis, Nat. Phys. 4, 287 (2008).

[31] L. de' Medici, X. Wang, M. Capone, and A. J. Millis, Phys. Rev. B 80, 054501 (2009).
[32] C. Weber, K. Haule, and G. Kotliar, Phys. Rev. B 78, 134519 (2008).

[33] Q. Yin, A. Gordienko, X. Wan, and S. Y. Savrasov, Phys. Rev. Lett. 100, 066406 (2008).

[34] P. Hansmann, N. Parragh, A. Toschi, G. Sangiovanni, and K. Held, New J. Phys. 16, 033009 (2014).

[35] H. Ebrahimnejad, G. A. Sawatzky, and M. Berciu, Nat. Phys. 10, 951 (2014).

[36] D. Bauernfeind, R. Triebl, M. Zingl, M. Aichhorn, and H. G. Evertz, Phys. Rev. B 97, 115156 (2018).

[37] P. Werner, R. Sakuma, F. Nilsson, and F. Aryasetiawan, Phys. Rev. B 91, 125142 (2015).

[38] P. Werner and M. Casula, J. Phys.s: Condens. Matter 28, 383001 (2016).

[39] J. D. Rameau, S. Freutel, L. Rettig, I. Avigo, M. Ligges, Y. Yoshida, H. Eisaki, J. Schneeloch, R. D. Zhong, Z. J. Xu, G. D. Gu, P. D. Johnson, and U. Bovensiepen, Phys. Rev. B 89, 115115 (2014).

[40] K. Matsuda, I. Hirabayashi, K. Kawamoto, T. Nabatame, T. Tokizaki, and A. Nakamura, Phys. Rev. B 50, 4097 (1994).

[41] H. Okamoto, T. Miyagoe, K. Kobayashi, H. Uemura, H. Nishioka, H. Matsuzaki, A. Sawa, and Y. Tokura, Phys. Rev. B 82, 060513(R) (2010).

[42] H. Okamoto, T. Miyagoe, K. Kobayashi, H. Uemura, H. Nishioka, H. Matsuzaki, A. Sawa, and Y. Tokura, Phys. Rev. B 83, 125102 (2011).

[43] F. Novelli, G. De Filippis, V. Cataudella, M. Esposito, I. Vergara, F. Cilento, E. Sindici, A. Amaricci, C. Giannetti, D. Prabhakaran et al., Nat. Commun. 5, 5112 (2014).

[44] F. Cilento, G. Manzoni, A. Sterzi, S. Peli, A. Ronchi, A. Crepaldi, F. Boschini, C. Cacho, R. Chapman, E. Springate, H. Eisaki, M. Greven, M. Berciu, A. F. Kemper, A. Damascelli, M. Capone, C. Giannetti, and F. Parmigiani, Sci. Adv. 4, eaar1998 (2018).

[45] B. Mansart, J. Lorenzana, A. Mann, A. Odeh, M. Scarongella, M. Chergui, and F. Carbone, Proc. Natl. Acad. Sci. USA 110, 4539 (2013).

[46] C. Giannetti, F. Cilento, S. Dal Conte, G. Coslovich, G. Ferrini, H. Molegraaf, M. Raichle, R. Liang, H. Eisaki, M. Greven et al., Nat. Commun. 2, 353 (2011).

[47] V. Emery, Phys. Rev. Lett. 58, 2794 (1987).

[48] S. Biermann, F. Aryasetiawan, and A. Georges, Phys. Rev. Lett. 90, 086402 (2003).

[49] T. Ayral, S. Biermann, and P. Werner, Phys. Rev. B 87, 125149 (2013).

[50] L. Huang, T. Ayral, S. Biermann, and P. Werner, Phys. Rev. B 90, 195114 (2014).

[51] P. Sun and G. Kotliar, Phys. Rev. B 66, 085120 (2002).

[52] R. Loudon, The Quantum Theory of Light (Oxford University Press, Oxford, 2000).

[53] D. Golez, M. Eckstein, and P. Werner, arXiv:1903.08713.

[54] We have checked that the results are not qualitatively dependent on the value of $D$.

[55] F. C. Zhang and T. M. Rice, Phys. Rev. B 37, 3759 (1988).

[56] T. Ayral, S. Biermann, P. Werner, and L. Boehnke, Phys. Rev. B 95, 245130 (2017).

[57] R. Sensarma, D. Pekker, E. Altman, E. Demler, N. Strohmaier, D. Greif, R. Jördens, L. Tarruell, H. Moritz, and T. Esslinger, Phys. Rev. B 82, 224302 (2010). 
[58] Z. Lenarčič and P. Prelovšek, Phys. Rev. B 90, 235136 (2014).

[59] P. Werner, K. Held, and M. Eckstein, Phys. Rev. B 90, 235102 (2014).

[60] M. Sandri and M. Fabrizio, Phys. Rev. B 91, 115102 (2015).

[61] The factor of 2 originates from the number of nearest-neighbor $p$ orbitals and we have used the conservation of the total charge $\Delta n_{d}=-\left(\Delta n_{p_{x}}+\Delta n_{p_{y}}\right)$.
[62] We have checked that for high-frequency excitations the shape of the spectra after the pulse is essentially the same.

[63] C. Giannetti, M. Capone, D. Fausti, M. Fabrizio, F. Parmigiani, and D. Mihailovic, Adv. Phys. 65, 58 (2016).

[64] L. Boehnke, F. Nilsson, F. Aryasetiawan, and P. Werner, Phys. Rev. B 94, 201106(R) (2016).

[65] F. Nilsson, L. Boehnke, P. Werner, and F. Aryasetiawan, Phys. Rev. Mater. 1, 043803 (2017). 